\title{
TEXTE MÉDIÉVAL, CONTEXTE ET PRAGMATIQUE LINGUISTIQUE
}

\author{
Malinka VeLINOVA \\ Université de Sofia et Université Paris-Sorbonne
}

\begin{abstract}
En): In recent years, the application of contemporary linguistics achievements, especially of the pragmatic approach, has proved itself particularly fruitful in the study of the enunciative and narrative structure of medieval literary works. In the present paper we suggest implementing that approach, which takes into account the discursive context, or co-text, as well as the situational context within which the speech act occurs, in the study of the relative clause in Old French. With this aim, we shall try to outline first the main stages in the evolution of the context concept in linguistics, then to determine the relevant elements in the context of the medieval text. In the conditions of enunciation in praesentia, which is the case with the enunciation of literary works in the Middle Ages, it is most likely that extralinguistic factors play a very important role in the communication of the literary message. Analysing the texts, we shall pay particular attention to the impact of the material, or physical, component of the utterance, that is, the vocalisation of texts during an oral performance (reinforced by gesture, mimic and intonation) or during a public reading of a written work (more or less limited in number). Using the example of the relative clause, we shall check the efficiency of applying the notion of the two types of context (linguistic and extralinguistic) in solving syntactic and pronominal ambiguities, trying to determine their role in the interpretation of texts by the medieval recipient as well as by the contemporary reader.
\end{abstract}

Keywords (En): Medieval French literature; enunciation in praesentia; (linguistic and extralinguistic) context; linguistic pragmatics; relative clause.

Mots-clés (Fr): littérature médiévale française; énonciation in praesentia; contexte (linguistique et extralinguistique); pragmatique linguistique ; proposition relative.

\section{Introduction}

Au cours de ces deux ou trois dernières décennies, on voit apparaître et prendre de l'essor une nouvelle tendance dans l'étude de la langue et de la syntaxe médiévales (cf. PerRet, 1988; Fleischman, 1990b ; MARNeTte, 1998), qui consiste à prendre en considération les facteurs énonciatifs et pragmatiques dans l'organisation discursive des textes médiévaux. Tout en empruntant cette voie déjà frayée, nous allons, pour notre part, montrer comment on peut étudier la syntaxe de la langue médiévale à partir des acquis dans le domaine de l'étude pragmatique des textes médiévaux, en nous appuyant sur les études qui ont pris de l'essor, elles aussi, depuis les années 1990 (cf. KoCH, 1993 ; OESTERREICHER, 1997 ; KOCH, OESTERREICHER, 2001 et 2007) et qui traitent des degrés d'oralité, d'oralisation et de scripturalité des textes médiévaux, de leur production, transmission, diffusion et réception.

Pour ce faire, nous suivrons essentiellement l'approche proposée par FLEISCHMAN (1990b : 3), qui envisage aussi bien le contexte discursif, ou le cotexte, que le contexte situationnel dans lequel l'acte de discours a lieu. Ainsi, la notion centrale pour nous sera celle de contexte, dont on va, dans un premier temps, essayer d'esquisser rapidement certaines caractéristiques essentielles générales pour pouvoir ensuite passer à l'examen de ses éléments constitutifs quant aux textes médiévaux. Nous espérons contribuer à combler de cette manière une certaine lacune que présentent, nous semble-t-il, les écrits sur le contexte 
d'une part et ceux sur la syntaxe médiévale d'autre part - à savoir négliger, jusqu'à récemment, le fait que l'impact de l'extralinguistique soit beaucoup plus considérable dans les conditions de production et de diffusion orales.

Ce sera à travers l'exemple de la relative que nous essaierons, dans un deuxième temps, de vérifier la validité de l'application de différents éléments et aspects des deux types de contextes (linguistique et extralinguistique), en particulier dans la résolution des cas d'ambiguïté syntaxique et/ou pronominale.

\section{La notion de contexte. La pragmatique du discours et la pragmatique situationnelle appliquées à l'étude des textes médiévaux}

Comme l'a noté KLEIBER (1994), entre autres, la notion de contexte employée en linguistique est assez ambiguë, floue et imprécise, et l'on y recourt chaque fois qu'un élément quelconque fait défaut pour l'analyse sémantique "proprement » linguistique, cette dernière étant basée sur le postulat que la langue est un code. Nous n'entrerons pas ici dans le détail pour ce qui est des divergences théoriques au sein des différents courants et thèses en pragmatique linguistique, notre objectif étant de montrer qu'il y a une certaine part d'influence du contexte ("général ») sur l'interprétation de certains traits spécifiques syntaxiques ou discursifs des textes au Moyen Âge, influence qui ne saurait être négligée ni du point de vue de l'auditeur médiéval ni du point de vue de l'analyste et du lecteur moderne.

Dans le contexte de la littérature médiévale, la pragmatique du discours s'avère, semble-t-il, malgré l'apparente contradiction entre démarche scientifique et spécificité de l'objet d'étude, comme l'approche la plus appropriée à l'étude des mécanismes de structuration narrative dans les textes. Nous suivrons dans ce qui suit la position théorique de MARNETTE :

«Par "contexte", il faut entendre ici le moment et le lieu où les énoncés sont proférés, le rôle social des participants, les règles de politesse qui régissent la situation d'énonciation, etc. » (MARNETTE, $1998: 22$ )

L'auteur doit beaucoup aux travaux de FLEISCHMAN, dont l'un des plus grands mérites est sans doute celui d'avoir résolument introduit l'approche pragmatique dans les analyses des textes anciens romans. Quant à l'objet d'étude de la pragmatique du discours vs celui de la pragmatique situationnelle, FLEISCHMAN pose que la pragmatique du discours étudie l'organisation des textes comme des ensembles cohérents, tandis que la pragmatique situationnelle s'intéresse aux différents aspects du cadre extratextuel du discours (cf. FlEISCHMAN, 1990b : 3). C'est notamment à cause de cette possibilité d'application conjointe de ces deux aspects de la pragmatique que l'approche choisie par FLEISCHMAN s'inscrit parfaitement comme outil dans l'étude de la littérature orale. Et si l'auteur critique l'approche traditionnelle du texte médiéval, c'est parce qu'on l'étudie comme si c'était de la fiction moderne, sans prendre en considération le fait que dans les situations orales la signification est à chercher non pas dans le texte mais dans le contexte (cf. FLEISCHMAN, 1990b : 9).

Or, quand il s'agit des différents problèmes que posent les relations entre texte et contexte, ou les indices et les mécanismes de contextualisation, il convient de 
signaler, comme l'a fait KERBRAT-ORECCHIONI, la distinction entre les discours naturels et les discours littéraires (d'autant plus qu'il s'agit dans notre cas de corpus presque exclusivement formés de textes littéraires) :

«Dans le cas des discours naturels : l'analyste est un récepteur non destinataire, c'est-àdire qu'il traite un objet qui ne lui est en rien destiné [...] ;

Dans le cas du discours littéraire au contraire : l'analyste n'est qu'un destinataire parmi d'autres, d'un texte où les informations décisives pour sa compréhension sont en principe toutes fournies par l'auteur - son équipement descriptif est donc dans ce cas beaucoup plus complet. » (KERBRAT-ORECCHIONI, $1996: 47-48$ )

S'agissant du Moyen Âge, les choses se compliquent un peu : le destinataire d'une œuvre littéraire qui lui est contemporaine et qui est lue, interprétée, chantée devant lui en temps réel est en même temps un destinataire direct - présent sur le lieu de la performance orale ou de la lecture publique à voix haute (dans la plupart des cas). Le lecteur et l'analyste modernes se trouvent donc être éloignés d'un degré encore de l'énonciation du texte. Ils sont à la fois des destinataires parmi d'autres de l'œuvre et des destinataires qui doivent prendre en considération plusieurs facteurs jouant dans la situation d'énonciation médiévale, sur laquelle on ne peut aujourd'hui que faire des conjectures, plus ou moins plausibles, bien sûr.

\section{Le contexte situationnel du texte médiéval}

Il nous semble qu'il règne dans la littérature sur le sujet une confusion, plus ou moins importante, malgré la distinction apparente, entre les termes «contexte», « contexte extralinguistique », « contexte de production», «contexte de situation » même et «situation » :

«Entre contexte et situation, la distinction est a priori assez claire. Situation est de l'ordre de l'extralinguistique, du circonstanciel et du donné, et contexte de l'ordre du verbal et du construit, le sens se donnant à l'intersection de ces deux sphères du monde et de la langue. » (FRANCKEL, $2006: 51$ )

Quant au cotexte, pour FRANCKEL (2006: 52), il représente le contexte « immédiat », relatif à un mot ou à une séquence, et s'oppose donc au contexte «large », ou le contexte tout simplement, lequel pourtant peut s'apparenter à situation, même s'il est de l'ordre du textuel. Pour ce qui est du contexte, au sens le plus large du terme, c'est-à-dire la notion générale englobant tous les types de contexte, équivaudrait plutôt au mot «situation»selon ÉLUERD (1985: 13); l'auteur parle dans ce cas de « contexte ordinaire mondain ».

Il nous semble donc pertinent, afin d'éviter toute confusion éventuelle, d'employer le terme de «contexte situationnel» au sens de «situation (générale d'énonciation) », et le terme de «contexte linguistique », en précisant, là où ce sera nécessaire, s'il s'agit du contexte linguistique immédiat, ou cotexte, ou bien du contexte large, ainsi qu'en en déterminant les dimensions et la portée. 


\subsection{Caractéristiques générales : énonciation in praesentia, «oralité » ou « vocalité »}

Pour ce qui est du texte médiéval, nous nous intéresserons donc au contexte situationnel de l'œuvre orale ou oralisée. L'oralité du Moyen Âge devrait être comprise au sens où l'écrit est dicté et la lecture se fait à voix haute (cf. FLEISCHMAN, 1990a), la lecture solitaire ou à voix basse se faisant encore très rare (cf. PERRET, 2006).

La thèse de la diffusion orale des textes littéraires au Moyen Âge ne date pas de ces dernières années - elle est proposée par RYCHNER (1955), en ce qui concerne en particulier les chansons de geste françaises. C'est chez les anthropologues, ethnographes et historiens anglo-saxons surtout que l'on peut trouver des études beaucoup plus intenses et nombreuses concernant les rapports oralité/scripturalité ou littératie ${ }^{1}$.

Selon ZUMTHOR (1987 : 22), presque tous les textes qui nous sont parvenus du $\mathrm{X}^{\mathrm{e}}$ au XIV ${ }^{\mathrm{e}}$ siècle se caractérisent par un « transit vocal », qui était le seul moyen de leur réalisation et de leur socialisation. Le médiéviste inclut dans sa thèse les genres suivants: les chansons, les récits et déclamations de tout genre, les chroniques ; quant au roman, il y fait l'objet d'un examen à part.

«Que le texte ait été ou non composé par écrit importe, certes, parfois considérablement à son économie interne et à sa grammaire. Mais qu'il soit reçu par lecture individuelle directe, ou par audition ou spectacle, modifie profondément son effet sur le récepteur, donc sa signifiance. Cela reste vrai de la forme atténuée de performance, que constituerait une lecture publique faite par un interprète assis, ou même debout, devant son lutrin. » (ZUMTHOR, 1987 : 24)

Le terme d' " oralité » s'applique donc essentiellement aux textes destinés à la «performance» orale, d'après le mot de ZUMTHOR ${ }^{2}$. Selon KOCH (1993), on a affaire dans le cas des plus anciennes œuvres françaises, et romanes en général, à une «scripturalité à destin vocal ${ }^{3}$ », embrassant la production littéraire, qu'elle repose ou non sur des traditions discursives latines, et à une « oralité élaborée » au sein de cette même catégorie, lorsqu'il s'agit en particulier de la poésie orale profane (à savoir les poèmes épiques). KoCH et OESTERREICHER $\left(2007^{4}\right.$ ) soulignent la distinction fondamentale entre l'aspect conceptionnel et l'aspect médial de la production sous forme de texte. L'aspect conceptionnel relève soit de l'immédiat communicatif (l'oralité), soit de la distance communicative (la scripturalité). L'aspect médial a trait à la réalisation du texte par l'intermédiaire du code phonique ou par l'intermédiaire du code graphique. Et quant aux stratégies communicatives de l'immédiat et de la distance, elles «impliquent deux types complètement différents de discursivité linguistique» (KOCH, OESTERREICHER, $2001: 592)$ :

\footnotetext{
${ }^{1}$ Cf. Finnegan (1977), Ong (1982), Coleman (1996), entre autres.

${ }^{2}$ Celui d' «auralité » (aurality, en anglais) s'applique aux textes destinés à la lecture publique (cf. COLEMAN, 1996).

${ }^{3}$ L'expression est empruntée à ZUMTHOR, qui oppose «le texte [...] à destin vocal » au «texte proposé à la lecture » $(1987: 213)$.

${ }^{4}$ Pour la traduction espagnole de l'édition en allemand, cette dernière parue en 1990.
} 
«[...] dans l'immédiat communicatif, le sujet parlant opère une contextualisation multiple, en s'appuyant sur tous les contextes possibles ("co-texte" linguistique ; phénomènes suprasegmentaux ; gestes, mimique, etc. ; contextes situationnel, pragmatique, épistémique, etc. [...]) ; la distance communicative, par contre, réduit considérablement l'accessibilité de certains contextes [...]. » (KOCH, OESTERREICHER $2001: 592)$

Suivant ce modèle et les thèses de ZUMTHOR, les plus anciennes œuvres en langue française, comme les chansons de geste et les vies de saints, relèveraient de la scripturalité à destin vocal (ou la distance communicative réalisée par le code phonique), tandis que les autres genres, plus tardifs (le roman en vers en particulier), seraient censés tenir plutôt de la scripturalité à proprement parler, ou de la distance communicative. Or, selon VITZ (1999), les romans français en vers octosyllabiques seraient aussi à considérer en tant qu'œuvres procédant de l'oralité et de la performance orale. En effet, il semble que le texte médiéval ait été l'objet d'un traitement spécifique qui relève de l'oral et de l'écrit dans le même temps et qui ait été régi par des tensions diverses et variables, en fonction de l'époque de composition et du genre de l'œuvre, vers les deux pôles de la communication.

\subsection{Les éléments du contexte situationnel des œuvres orales ou oralisées}

Quels sont donc les éléments pertinents concrets du contexte situationnel du texte médiéval, dans les conditions de l'énonciation in praesentia?

Il s'agit, en premier lieu, de la communication par l'intermédiaire de la voix, de la transmission ou diffusion orale de l'œuvre, en présence physique des participants dans l'acte de «réalisation » de l'œuvre - l'interprète ou le lecteur et les auditeurs ou le public, et ce sans tenir compte de la manière dont l'œuvre a été composée (oralement ou par écrit): qu'il s'agisse d'une composition orale ou écrite, les poèmes épiques sont tous communiqués lors de la performance, ce qui ne veut pas dire qu'ils relèvent de l'immédiat communicatif (cf. OESTERREICHER, 1997 : 207).

D'autre part, même si les auditeurs/spectateurs n'ont pas la possibilité de revenir en arrière pour relire les passages obscurs et le cotexte qui les précède possibilité dont peut seul se servir le lecteur pratiquant la lecture solitaire, qu'elle soit à voix haute ou à voix basse -, ils ont toutefois une autre possibilité, celle d'interpeller l'interprète (cf. PERRET, 2006). Il se peut même qu'à cette possibilité d'interaction entre interprète et public viennent s'ajouter les intonations et la gestuelle désambiguïsantes, comme le fait justement remarquer PERRET à propos de la labilité référentielle dans les œuvres médiévales :

«[...] l'interlocuteur peut toujours réagir à l'équivoque et la faire préciser. Si elle n'est pas exclue de l'écrit, c'est dû aux conditions de transmission du texte, récité ou lu en public, une énonciation in praesentia, avec l'emploi d'intonations et surtout d'une gestuelle désambiguïsantes. » (PERRET, $2006: 26$ )

Et même si la performance elle-même est strictement monologique, il existe donc une sorte de coopération entre les auditeurs/spectateurs et l'interprète, ceuxlà faisant des commentaires, donnant libre cours à leurs réactions émotionnelles, voire encourageant celui-ci (cf. OESTERREICHER, 1997 : 208). 
On n'oubliera pas non plus les formules stéréotypées, qui sont étroitement liées aux mécanismes mémoriels (mnémotechniques) chez l'interprète et le public à la fois, même s'il ne s'agit pas nécessairement de composition orale des textes (comme le veut la thèse de Parry et Lord), sauf peut-être dans le cas des premiers poèmes épiques et fabliaux.

\section{La relative et le contexte linguistique et extralinguistique : les cas de disjonction du relatif de son antécédent ${ }^{5}$}

\subsection{Les relatives disjointes en ancien français et en français moderne}

Selon R.-L. WAGNER, les cas de disjonction du relatif de son antécédent pourraient être portés «au compte des conventions rédactionnelles diverses qui confèrent "un style" à l'ancien français » (1974: 66), puisqu'on en trouve plusieurs exemples tant en poésie qu'en prose et que le phénomène ne s'est pas perpétué en moyen français. SALVAN (2009 : 62) souligne, pour ce qui est de la relative disjointe en français contemporain, qu'il s'agit là d'une disjonction « marquée », d'emploi stylistique ; or, ce n'est pas toujours le cas lors de l'emploi de la distance entre le relatif et l'antécédent en ancien français. En ce qui concerne la syntaxe de la relative en ancien français, on doit avoir en vue que :

« [s]i la proposition principale ne contient qu'un syntagme nominal et un syntagme verbal, dans une langue typiquement à verbe second, la subordonnée relative subséquente est séparée de son antécédent. »(BURIDANT, 2000 : 581)

FOURNIER a montré qu'une « approche mémorielle cognitive, qui se fonde sur le degré de saillance du référent dans la mémoire immédiate des co-énonciateurs et fait entrer en jeu la notion de hiérarchie de saillance topicale » (1998: 192), se révèle mieux adaptée à la construction et à l'interprétation des énoncés en langue classique et apte, par conséquent, à réduire les ambiguïtés qu' on y relève du fait de la distance parfois trop grande entre l'anaphore et son référent. Nous allons essayer, pour notre part, de vérifier dans quelle mesure cette approche peut paraître appropriée à la situation de la langue médiévale.

En raison de la spécificité de l'objet étudié, nous avons procédé à un dépouillement manuel des quelques textes des $\mathrm{XII}^{\mathrm{e}}$ et $\mathrm{XIII}^{\mathrm{e}}$ siècles qui constituent, à cette étape, notre corpus, et qui sont de forme (vers, prose) et de genres différents (chanson de geste, vie de saint, roman courtois, lai, chronique). Ne prétendant donc nullement à l'exhaustivité, nous ne nous arrêterons ici que sur quelques cas significatifs du phénomène en question.

\subsection{Le principe de saillance topicale}

Dans les exemples (1) à (7), tous en vers, l'ambiguïté virtuelle se trouve levée par la dominance du principe de saillance sur celui de proximité - le déterminé l'emporte sur le déterminant, comme en (1), le sujet sur le complément direct ou prépositionnel (cf. FOURNIER, 1998 : 197), que ce dernier soit pronominal, comme

\footnotetext{
${ }^{5}$ Cf. aussi notre article (Velinova, 2009) sur les différents cas d'ambiguïté dans l'emploi du relatif en français médiéval, dont nous reprenons ici certains exemples et analyses.
} 
en (3) et (4), ou nominal, comme dans les autres exemples. Le sujet, lui aussi, peut être pronominal dans certains cas - moins nombreux pourtant, selon nos dépouillements -, comme en (6).

(1) Dist li cuens : « Damme, ci a grant mesprison.

Ja voz demande li fors rois d'Arragon

Et d'Espolice Girars li fiuls Othon',

Qui mainne an ost plus de mil compaignons. [...] » (Ami et Amile, v. 631-634)

(2) Molt erent tout en l'ost haitié,

cil a cheval et cil a pié,

quant uns mes vint devant le rei,

qui li nounça par grant desrei : [...]. (Roman de Thèbes, v. 2761-2764)

(3) Charlemaine l'ad eguardé,

$k i$ en la chapele l'atent [...]. (G. de Berneville, Vie de saint Gilles, v. 3084-3085)

(4) Et cil a tant a esperon

Toutes voies Cligés chacié,

Touz armez, le hiaume lacié,

Que Cligés le voit seul venir,

Qui onc ne volt apartenir

A recreant n'a cuer failli. (Chr. de Troyes, Cligès, v. 3428-3433)

(5) Li rois li rendi ses salus,

Qui de respondre ne fu mus,

Et se li a dit : « Descendés. » (R. de Beaujeu, Bel Inconnu, v. 79-81)

(6) Yseut est en sa chambre entree.

Brengain la vit decoloree ;

Bien sout que ele avoit oï

Tel rien dont out le cuer marri,

Qui si muoit et palisoit [...]. (Béroul, Tristan, v. 339-343)

(7) Tant ont li messaigier erré

qu'il vindrent a la cité

que Enea envoia querre

l'estre de lui et de la terre. (Roman d'Énéas, v. 516-519)

L'exemple en (7) présente un cas plus intéressant du point de vue syntaxique, le sujet, qui est l'antécédent de la relative, étant repris par une autre anaphore, le pronom personnel il $\left(3^{\mathrm{e}}\right.$ personne du pluriel), avant d'être repris par le relatif complément que. C'est la troisième fois que le long des 200-300 vers qui précèdent ceux en (7) il est question des messagers envoyés par Énéas. Les vers 518-519 apparaissent donc comme une simple formule de rappel. Il n'y a donc pas d'ambiguïté quant à l'antécédent de que, sauf peut-être pour l'auditeur, ou le lecteur, très distrait (la cité aurait bien pu être l'antécédent de que, et l'objet de querre par conséquent, si la relative se terminait avec le vers 518). Ou c'est justement dans le but de rappeler au public de quels personnages exactement il

\footnotetext{
${ }^{6}$ Nous mettons, dans nos exemples, en italique le pronom relatif et son antécédent ; nous marquons en caractères gras le deuxième antécédent possible et soulignons le troisième, s'il y en a.
} 
s'agit qu'est réintroduit le motif : la relative n'aurait dans ce cas qu'une fonction mnémotechnique, et ce serait donc le contexte linguistique large qui entre en jeu ici.

L'application de l'approche mémorielle de l'anaphore s'avère donc tout aussi pertinente, voire plus, dans le cas de l'ancienne langue que dans celui de la langue classique. Peut-être est-il vrai que, s'agissant de l'anaphore, dans les conditions d'une oralité prédominante, on s'attendrait plutôt à l'action du principe de proximité, du fait que le public auquel est destinée l'œuvre n'a pas dans la plupart des cas la possibilité de revenir en arrière pour relire le passage ambigu. Et ce serait pourtant justement à cause de cette oralité, ou plutôt vocalité, que les interlocuteurs, ou destinataires, en l'occurrence le public, auraient pris l'habitude de bien identifier les éléments saillants pour pouvoir ensuite reconnaitre sans faille les référents des relatives éloignées. La méthode mnémotechnique n'est-elle pas mieux appropriée aux cultures orales, la mémoire, la mémorisation faisant partie intégrante du savoir, de sa transmission ${ }^{7}$ ? C'est justement le contexte situationnel qui conditionne une pareille approche.

Dans les exemples relevés ci-dessous, les cas les plus nombreux, sont ceux où l'on retrouve la relative explicative, comme en (1), (3) et (4), ainsi qu'en (5), (6) et (7), mais la relative en (5) et (6) a une valeur circonstancielle, respectivement causale et consécutive; nous y reviendrons un peu plus loin. Dans nos exemples ici, il n'y a aucune occurrence de relative restrictive, et il n'y en a qu'une seule de relative « de postériorité », d'après le terme de ROTHENBERG (1972: 189), en (2). C'est ce dernier type que BRUNNER (1981: 15) appelle «relative narrative », compte tenu de son rôle, qui est de faire progresser le récit; relative dont les origines sont à chercher sans doute, selon lui, dans la relative «de liaison» du latin.

Toutefois, on pourrait rapprocher l'énoncé en (3) en particulier de la structure suivante «il le voit qui arrive » (ou, plus précisément en l'occurrence, «il le voit qui $l^{\prime}$ attend $»^{8}$ ), et dans ce cas, il faudrait recourir au contexte linguistique (de gauche ou de droite) pour que l'ambiguité soit levée. Ceci est valable également pour la relative en (4). Or, il ne s'agirait là que d'une possibilité d'interprétation ambiguë pour le lecteur contemporain.

Dans la prose du XIII ${ }^{\mathrm{e}}$ siècle, apparaît déjà un ordre des mots plus « régulier », en ce qui concerne en particulier la place de l'antécédent et du relatif (cf. (8) et (9)) : voir $+\mathrm{SN}$ objet $+q u i$, le relatif se rapportant à l'objet et le suivant sans aucune distance, avant le figement (la grammaticalisation) de la construction de type «il le voit qui arrive», processus qui commence vers le milieu du XIV ${ }^{\mathrm{e}}$ siècle, d'après du moins les recherches préliminaires que nous avons menées dans le DMF.

(8) Qant Lancelos vit son compaignon qui devant lui fu portez a terre si durement, si en est mult corociez. (Mort du roi Arthur, § 12, 1. 1-2)

\footnotetext{
${ }^{7}$ Cf. CARRUTHERS (2008).

${ }^{8}$ C'est-à-dire les relatives après les verbes de perception, que certains appellent «prédicatives » et d'autres, notamment ROTHENBERG (1979), «attributives », et qui présenteraient une construction spécifique de la relative anaphorique à côté des deux types traditionnels (cf. PREBENSEN, 1982).
} 
(9) Ne demora gaires que li autres chevaliers vint après celui tot contraval la riviere, si s'arestut tres desus l'eive es prez sor la riviere et vit fames qi lavoient dras, si lor demande : [...]. (Lancelot du Lac, f. 84d)

\subsection{Cas où le principe de saillance n'apporte pas (à lui seul) de désambiguïsation}

Or, il y a des cas en ancien français, comme dans ce qui suit, où la saillance topicale peut paraître un critère inapte à lever l'ambiguïté, ou du moins insuffisant, comme en (10), pour la résoudre.

(10) La dame lieve, si la prent,

Ariere va hastivement,

Dedenz la buche a la pucele

meteit la flur $k i$ tant fu bele. (M. de France, Éliduc, v. 1059-1062)

La relative ici se rapporterait plutôt à flur qu'à pucele ou buche. Il n'y a aucun indice syntaxique en terme de saillance, dans ce cas, qui puisse lever l'ambiguïté : le seul indice dont on dispose est de nature sémantico-contextuelle - le narrateur a déjà souligné la beauté de la jeune fille dont il s'agit. La relative qui tant fut bele requiert alors une valeur de formule (rappelant celles des chansons de geste); elle ne sert d'ailleurs que de remplir le vers et d'assurer la rime en -ele (c'est-à-dire qu'elle n'a pas de rôle sémantique particulier, son rôle n'est que stylistique, esthétique). Dans ce cas, paraît complètement valable l'affirmation de KUNSTMANN que parfois «seule la prise en compte du référent permet de saisir l'antécédent » (1990 : 238).

(11) Issi la pucele delaie,

De sa parole molt $l$ 'apeie,

Qui de tot cuide estre s'amie. (R. de Beaujeu, Bel Inconnu, v. 3415-3417)

La désambiguïsation en (11) ne vient, du point de vue linguistique, que du genre grammatical du mot amie, ainsi que du fait que l'antécédent doit être animé ; ni le sujet nominal, ni d'ailleurs un quelconque sujet pronominal, n'apparaît dans cet énoncé. La relative dans cet exemple n'a-t-elle pas une valeur circonstancielle ? Si l'on repère bien des occurrences de qui introduisant une proposition non déterminative circonstancielle (dans les textes épiques en particulier, pour ce qui est surtout de la valeur causale (cf. BERTIN, 1997 : 124), cf. aussi nos exemples (5) et (6)), ne pourrait-il pas s'agir ici d'une relative à valeur consécutive, qui équivaudrait à «si bien qu'elle... »; ou serait-il question d'une causale, «puisqu'elle croit, de tout cela, qu'il l'aime»? Ainsi, même si l'antécédent et le référent se trouvent être bien identifiés, l'ambiguïté quant à la valeur logique de la relative persiste.

C'est surtout dans les textes en prose que l'on rencontre des suites de relatives, parfois combinées à d'autres subordonnées, les séquences étant le plus souvent juxtaposées ${ }^{9}$. Chez Villehardouin en particulier, la juxtaposition de plusieurs

\footnotetext{
${ }^{9}$ Cette constatation n'a rien d'étonnant, le discours poétique, surtout épique, produisant des énoncés qui s'inscrivent, dans la majorité des cas, dans la structure d'un seul vers; d'où les constructions
} 
relatives peut entraîner facilement l'ambiguïté - du moins pour le lecteur contemporain - en ce qui concerne le rattachement de la relative, comme en (12) et (13) :

(12) La contesse remest, sa fame, qui Blanche avoit non, moult bele et moult bone, et iert fille le roy de Navarre, qui avoit de lui une fille, et estoit grosse d'un filz. (Villehardouin, $\mathrm{La}$ Conquête de Constantinople, §37)

(13) Et li emperieres Morchufles ne tarda gueres qu'il prist une cité qui estoit en la merci l'empereeur, que l'en apeloit le Churlot, si le prist et roba, et prist quanque il y trouva. (ibid., § 267)

L'ambiguïté, en l'occurrence, surtout en (13) (le texte de Villehardouin présente plusieurs exemples de ce type précisément), mais aussi en (12) jusqu'à l'étape de la lecture de de lui dans le contexte de droite immédiat, provient de la manière particulière de l'ancienne langue d'enchaîner les relatives :

«Quand une relative porte sur un groupe formé d'un nom ou d'un pronom suivi d'une relative, la deuxième relative n'est pas coordonnée à la première par et comme en FM. » (MENARD, 1994 : 94)

KUNSTMANN affirme, pour sa part, que l'ancien français « juxtapose librement deux relatives liées au même antécédent » (1990: 256). Ainsi a-t-on en (13) deux séries de deux relatives chacune, les premières relatives étant introduites par le pronom relatif qui, les secondes s'en passant. La lecture ambiguë de la deuxième série se trouve favorisée en outre par le pronom anaphorique lui, que l'on croirait référer à roy de Navarre. MARCOTTE (2000 : 14) souligne que cette structure est propre à l'ancienne étape de la langue, le moyen français faisant usage de la coordination, lorsqu' on a une suite hétérogène (de relative restrictive et de relative appositive), ce qui est le cas en (13). L'ancien français et les textes versifiés en particulier présentent parfois des cas de coordination singuliers, du fait de l'ordre des constituants dans la relative, comme en (14), où l'on a le relatif en deuxième position, tout comme en (15), sans qu'il y ait dans ce second cas deux ou plusieurs relatives coordonnées mais une seule :

(14) Dorment cum cil qui sunt lassét

E tanz perilz qui unt passéet. (Benedeit, Voyage de saint Brendan, v. 573-574)

(15) Respunt Judas : «Pres est li lius

As diables $u$ est li fius. [...]» (ibid., 1323-1324)

En (16), l'ordre des mots OVS désigne femme comme l'élément rhématique emphatisé, qui serait donc l'antécédent disjoint de dont, et le relatif aurait le sens de «à cause de qui » (en dehors du contexte, ou, si l'on veut, du sujet, et faisant abstraction de la dominance syntaxique de l'emphase, on pourrait aussi interpréter la relative comme restrictive, référant à li seneschals). Mais dont pourrait également avoir le sens de « ce dont» (c’est-à-dire «le fait que le sénéchal a pris

paratactiques propres à ce type de textes. Comme l'affirme MARCHELLO-NIZIA, « langue du vers et langue de la prose sont différentes, et dès l'origine » $(1978: 34)$. 
une épouse ») : l'ambiguïté se trouve donc maintenue, au cas où il n'y aurait pas d'intonation ou de gestuelle désambiguïsantes.

(16) Femme espuse ot li seneschals,

Dunt puis vint el païs granz mals. (M. de France, Équitan, v. 29-30)

C'est le cas aussi en (17) :

(17) $[\ldots]$ - Car tele amor a vostre fius a Blanceflour, cele fille vostre kaitive, ja tant come ele sera vive, l'amor de $l i$ ne cangera ne autre feme ne prendra, dont seroit forment ahontés de li tos nostres parentés. (R. d'Orbigny, Floire et Blanchefleur, v. 291-298)

Or, dans ce dernier cas l'interprétation avec «ce dont» semble de loin plus pertinente, vu la distance considérable entre l'antécédent (éventuel) Blanceflour et le relatif dont. Mais même si l'on interprète dont par «ce dont», on est à nouveau face à une ambiguïté, l'antécédent pouvant être aussi bien la séquence intégrale de l'énoncé qui précède que la séquence ne autre feme ne prendra.

Il y a des cas enfin, comme celui en (18), où la disjonction gêne l'interprétation de la relative - du point de vue de son type sémantique d'abord restrictive ou appositive -, mais aussi en ce qui concerne le choix du bon antécédent, ce qui est étroitement lié, en l'occurrence, à l'identification sémantique de la relative.

(18) L'ewe buillant fait aporter,

$u$ li seneschals dut entrer. (M. de France, Équitan, v. 281-282)

Il existe en effet deux possibilités dans ce cas : $1 / u$ employé sans antécédent se rapporte à l'endroit, à la chambre (l'adverbe la fait souvent défaut en ancien français, surtout dans les textes versifiés), mais le contexte linguistique (un peu plus large) de gauche indique que les personnages sont déjà là ; $2 /$ ewe est l'antécédent de $u$, et la relative est appositive ${ }^{10}$. Ce n'est que le recours au contexte extérieur à l'énoncé donné qui contribue à ce que l'ambiguïté soit levée (d'ailleurs, la virgule n'arriverait pas à être significative, sa présence étant sans doute le fait de l'éditeur moderne).

Les exemples (10) à (18) illustrent tous la nécessité de recourir aux spécificités du contexte situationnel de l'œuvre médiévale française, où entrent en jeu des procédés intonatifs ou gestuels de désambiguïsation.

\footnotetext{
${ }^{10}$ Il est vrai cependant que la lecture restrictive n'est point exclue dans ce cas (cf. WILMET, 1986 : 49-50), mais de telles analyses dépassent les problèmes que pose la relative disjointe en ancien français.
} 


\section{Conclusion}

La notion de contexte appliquée à l'étude des textes médiévaux apparaît donc plus complexe encore, en fonction de leur caractère oral, dont il ne reste aujourd'hui que des traces qui sont sans doute à repérer par les outils contemporains de la linguistique. Le contexte est donc vu ici, encore une fois, comme un ensemble d'éléments linguistiques et d'éléments extralinguistiques (dans la situation de performance orale ou dans celle de lecture publique), au sujet desquels nous ne saurions faire que des hypothèses, plus ou moins plausibles, sur la pertinence par rapport à la situation d'énonciation et la situation d'interprétation (contemporaine à l'œuvre, donc pour les auditeurs médiévaux). Nous avons essayé, dans le cadre d'un corpus assez restreint à cette étape, d'observer certains éléments pertinents du contexte dans le traitement ou l'appréhension de la relative dans les textes en ancien français.

En ce qui concerne en particulier les cas d'ambiguïté résultant de l'emploi de la relative disjointe en ancien français, nous avons observé certains exemples qui paraissent ambigus aussi bien pour le lecteur moderne que probablement pour l'auditeur médiéval. Dans une grande partie des exemples relevés dans notre corpus, on peut recourir au principe de saillance topicale afin que l'ambiguïté soit résolue; or, il s'est avéré que parfois l'application de ce principe paraît insuffisante, surtout pour les vers, ou lorsqu'il s'agit de l'emploi de formes polysémiques (comme dont). Il y a également des cas, comme en (3) et (4), où l'ambiguïté n'apparaîtrait en particulier qu'aux yeux du lecteur contemporain non averti, du fait du rapprochement possible entre les relatives que contiennent ces exemples et les relatives dites «attributives », de type «il le voit qui arrive». Dans certains cas, comme en (18), seuls le recours au contexte de gauche et l'interaction avec l'interprète peuvent lever l'ambiguïté. En (10), par exemple, le recours à des mécanismes grammaticaux et syntaxiques de levée de l'ambiguïté n'y réussira pas non plus ; c'est sans doute dans des cas pareils qu'intervient avec un effet désambiguïsant la mimo-posturo-gestualité, ou la possibilité d'interaction entre l'interprète et le public qu'offre la situation d'énonciation in praesentia. Et c'est justement dans ces derniers cas que l'hypothèse de PERRET à propos des raisons pragmatiques du maintien de la labilité référentielle en français médiéval se trouve entièrement validée.

Quant au lecteur contemporain, il doit prendre en considération aussi bien la situation de transmission orale des textes médiévaux que les spécificités syntaxiques de l'ancienne langue, c'est-à-dire les spécificités d'une langue et d'une syntaxe en cours de standardisation - processus qui ne commence, du moins pour ce qui est des occurrences de la relative dans le cadre de notre corpus, à se manifester de plus en plus nettement qu'avec les textes en prose. 


\section{BIBLIOGRAPHIE}

BERTIN Annie (1997), L'expression de la cause en ancien français, Genève, Droz. BRUNNER Jean-Jacques (1981), Ces relatives qui n'en sont pas, L'information grammaticale 8 , p. 12-16.

BURIDANT Claude (2000), Grammaire nouvelle de l'ancien français, Paris, SEDES.

CARRUTHERS Mary (2008), The Book of Memory: A Study of Memory in Medieval Culture, Cambridge, Cambridge University Press, $2^{\text {nd }}$ edition.

Coleman Joyce (1996), Public reading and the reading public in late medieval England and France, Cambridge, Cambridge University Press.

ÉLUERD Roland (1985), La pragmatique linguistique, Paris, Nathan.

FINNEGAN Ruth (1977), Oral Poetry. Its nature, significance and social context, Cambridge, Cambridge University Press.

FLEISCHMAN Suzanne (1990a), Philology, Linguistics, and the Discourse of the Medieval Text, Speculum 65, p. 19-37.

FLEISCHMAN Suzanne (1990b), Tense and Narrativity. From Medieval Performance to Modern Fiction, Austin, University of Texas Press.

FOURNIER Nathalie (1998), Norme et usage de l'anaphore pronominale en français classique: principe de proximité et principe de saillance du référent, in : BAUdry Janine, CARON Philippe (éds), Problèmes de cohésion syntaxique de 1550 à 1720, Limoges, PULIM, p. 191-214.

FRANCKEL Jean-Jacques (2006), Situation, contexte et valeur référentielle, Textes, contextes, Pratiques 129-130, p. 51-70.

KERBRAT-ORECCHIONI Catherine (1996), Texte et contexte, in : ScHMOLL Patrick (dir.), Contexte(s), Scolia 6, p. 39-60.

KLEIBER Georges (1994), Contexte, interprétation et mémoire : approche standard vs approche cognitive, Langue française 103, p. 9-22.

KoCH Peter (1993), Pour une typologie conceptionnelle et médiale des plus anciens documents/monuments des langues romanes, in : SELIG Maria et al. (éds), Le passage à l'écrit des langues romanes, Tübingen, Gunter Narr, p. 3981.

KOCH Peter, OESTERREICHER Wulf (2001), Langage parlé et langage écrit, Lexikon der Romanistischen Linguistik 2, Tübingen, Max Niemeyer, p. 584627.

KOCH Peter, OESTERREICHER Wulf (2007), Lengua hablada en la romania: español, francés, italiano, trad. esp., Madrid, Gredos.

KUNSTMANN Pierre (1990), Le relatif-interrogatif en ancien français, Genève, Droz.

MARCHELlO-NIZIA Christiane (1978), Un problème de linguistique textuelle : la classe des éléments joncteurs de propositions, in: MARTIN Robert (éd.), Études de syntaxe de moyen français, Paris, Klincksieck.

MARCOTTE Stéphane (2000), La coordination de relatives incidentes à un antécédent différent: un tour propre au moyen français ?, L'information grammaticale 87, p. 10-16. 
MARNETTE Sophie (1998), Narrateur et points de vue dans la littérature française médiévale. Une approche linguistique, Berne, Peter Lang.

MENARD Philippe (1994), Syntaxe de l'ancien français, Bordeaux, Bière, 4 éd.

OESTERREICHER Wulf (1997), Types of Orality in Text, in : BAKKER Egbert,

KaHAnE Ahuvia (éds), Written Voices, Spoken Signs. Tradition, Performance, and the Epic Style, Cambridge/London, Harvard University Press.

ONG Walter J. (1982), Orality and literacy: The technologizing of the word, London/New York, Methuen.

PERRET Michèle (1988), Le signe et la mention : adverbes embrayeurs CI, CA, LA, ILUEC en moyen français (XIV-XV $V^{\circ}$ siècles), Genève, Droz.

PERRET Michèle (2006), Ancien français : quelques spécificités d'une énonciation in praesentia, Langue française 149, p. 16-30.

PREBENSEN Henrik (1982), La proposition relative dite attributive, Revue romane 17, p. 98-117.

ROTHENBERG Mira (1972), Les propositions relatives adjointes en français, Bulletin de la Société de Linguistique de Paris 67, p. 175-213.

RothenBERG Mira (1979), Les propositions relatives prédicatives et attributives : problème de linguistique française, Bulletin de la Société de linguistique de Paris 74, p. 351-395.

RYCHNER Jean (1955), La chanson de geste. Essai sur l'art épique des jongleurs, Genève, Droz / Lille, Giard.

SALVAN Geneviève (2009), Le dialogisme dans les relatives disjointes, Langue française 163, p. 61-78.

VELINOVA Malinka (2009), L'ambiguïté dans l'emploi du relatif-interrogatif en français médiéval, in : AVANZI Mathieu et al. (éds), L'ambiguïté dans les sciences du langage, TRANEL 50, p. 19-35.

VITZ Evelyn B. (1999), Orality and Performance in Early French Romance, Cambridge, D. S. Brewer.

WAGNER Robert-Léon (1974), L’ancien français, Paris, Larousse.

WILMET Marc (1986), La détermination nominale, Paris, PUF.

ZUMTHOR Paul (1983), Introduction à la poésie orale, Paris, Le Seuil.

ZUMTHOR Paul (1987), La lettre et la voix, Paris, Le Seuil.

\section{CORPUS}

vers 1200 - Ami et Amile, éd. DemBowsKi Peter F. (1987), Paris, Champion.

début XII ${ }^{\mathrm{e}}$ s. - Benedeit, Le Voyage de saint Brendan, éd. SHORT Ian, MERRILEES

Brian (2006), Paris, Honoré Champion.

vers 1150 - Robert d'Orbigny, Le Conte de Floire et Blanchefleur, éd.

LECLANCHE Jean-Luc (2003), Paris, Honoré Champion.

vers 1150 - Le Roman de Thèbes, éd. MORA-LEBRUN Francine (1995), Paris,

LGF.

vers 1160 - Le Roman d'Énéas, éd. PETIT Aimé (1997), Paris, LGF.

vers 1170 - Lais de Marie de France, trad., prés. et annot. HARF-LANCNER

Laurence, éd. WARNKE Karl (1990), Paris, LGF. 
vers 1176 - Chrétien de Troyes, Cligès, in : Romans, éd. Mela Charles, Collet Olivier (1994), Paris, LGF.

1150-1190 - Béroul, Le Roman de Tristan, in: Tristan et Iseut: les poèmes français, la saga norroise, éd. LACROIX Daniel, WALTER Philippe (1989), Paris, LGF.

dernier tiers du XII ${ }^{\mathrm{e}}$ s. - Guillaume de Berneville, La Vie de saint Gilles, éd. LAURENT Françoise (2003), Paris, Honoré Champion.

début XIII s. - Renaud de Beaujeu, Le Bel Inconnu, éd. PERRET Michèle (2003), Paris, Honoré Champion.

début XIII ${ }^{\mathrm{e}}$ s. - Lancelot du Lac, prés., trad. et annot. MoSES François, d'après l'éd. de KENNEDY Elspeth (1991), Paris, LGF.

après 1207 - Geoffroy de Villehardouin, La Conquête de Constantinople, éd. DUFOURNET Jean (2004), Paris, Flammarion.

XIII ${ }^{\mathrm{e}}$ s. - La Mort du roi Arthur, éd. BAUMGARTNER Emmanuèle, DE MEdeIROS Marie-Thérèse (2007), Paris, Honoré Champion.

DMF : Dictionnaire du Moyen Français, version 2010. ATILF - CNRS \& Nancy Université. Site internet : http://www.atilf.fr/dmf 